\title{
The Role of Obesity in Sepsis Outcome among Critically Ill Patients: A Retrospective Cohort Analysis
}

\author{
Matthaios Papadimitriou-Olivgeris, ${ }^{1}$ Diamanto Aretha, ${ }^{2}$ Anastasia Zotou, ${ }^{2}$ \\ Kyriaki Koutsileou, ${ }^{2}$ Aikaterini Zbouki, ${ }^{2}$ Aikaterini Lefkaditi, ${ }^{2}$ Christina Sklavou, ${ }^{2}$ \\ Markos Marangos, ${ }^{1}$ and Fotini Fligou ${ }^{2}$ \\ ${ }^{1}$ Division of Infectious Diseases, School of Medicine, University of Patras, Rion, 26504 Patras, Greece \\ ${ }^{2}$ Department of Anaesthesiology and Intensive Care Medicine, School of Medicine, University of Patras, Rion, 26504 Patras, Greece
}

Correspondence should be addressed to Diamanto Aretha; adaretha@yahoo.gr

Received 28 April 2016; Revised 14 August 2016; Accepted 8 September 2016

Academic Editor: Flavia Prodam

Copyright (C) 2016 Matthaios Papadimitriou-Olivgeris et al. This is an open access article distributed under the Creative Commons Attribution License, which permits unrestricted use, distribution, and reproduction in any medium, provided the original work is properly cited.

\begin{abstract}
Background. The objective of this study was to assess the correlation between sepsis, obesity, and mortality of patients admitted to an Intensive Care Unit (ICU). Subjects and Methods. Data of all patients admitted to the ICU of a tertiary hospital during a 28month period were retrospectively analyzed and included in the study. Results. Of 834 patients included, 163 (19.5\%) were obese, while $25(3.0 \%)$ were morbidly obese. Number of comorbidities $(P<0.001)$, bloodstream infection $(P 0.033)$, and carbapenemaseproducing Klebsiella pneumoniae colonization during ICU stay $(P \quad 0.005)$ were significantly associated with obesity, while nonobese patients suffered more frequently from spontaneous intracranial hemorrhage $(P \quad 0.038)$. Total ICU mortality was $22.5 \%$. Increased mortality among obese ICU patients was observed. Sepsis was the main condition of admission for which obese patients had statistically lower survival than normal weight subjects ( $76.3 \%$ versus $43.7 \%$; $P$ 0.001). Mortality of septic patients upon admission was independently associated with SOFA score upon ICU admission $(P 0.003)$, obesity $(P 0.014)$, pneumonia $(P 0.038)$, and development of septic shock $(P$ 0.015). Conclusions. Our study revealed that sepsis upon ICU admission is adversely influenced by obesity but further studies are needed in order to assess the role of obesity in sepsis outcome.
\end{abstract}

\section{Introduction}

Obesity is attaining epidemic proportions in Europe especially Greece $[1,2]$. During the last decades obesity prevalence has increased significantly, especially among children and adolescents $[1,3]$. This increase that began in the $1980 \mathrm{~s}$ was attenuated over the last eight years [3]. Globally, an increase by $27.5 \%$ was observed in prevalence of overweight and obesity combined between 1980 and 2013 [3]. Nowadays, $71 \%$ of male and $51 \%$ of female adult Greeks are obese or overweight [3]. The prevalence of obesity was $28 \%$ and $26 \%$ among Greek men and women, respectively [4]. Obese patients are at increased risk of developing comorbidities, such as hypertension, coronary disease, chronic obstructive pulmonary disease, and diabetes [5]. In order to assess the presence of obesity the body mass index (BMI) is used and interpreted according to World Health Organization [6].
The relationship between obesity and mortality of critically ill patients remains unknown, since studies assessing the role of obesity in mortality among patients admitted to intensive care units (ICUs) show contradictory results $[7,8]$. Due to comorbidities, several studies reported higher mortality rates among obese critically ill patients. On the contrary, recent studies found lower mortality in obese than in normal weight ICU patients $[9,10]$, a phenomenon referred to as the "obesity paradox" [11].

This paradox was also observed in subgroups of critically ill patients, such as patients with septic shock or those with peritonitis $[12,13]$. The etiology for this paradox is not clear and can be due to selection bias in the study design or differences in patients' characteristics [11].

The aims of this study were to describe the epidemiology of obesity among critically ill patients hospitalized in a Greek 
ICU, to assess its effect on ICU mortality and to investigate the correlation between sepsis and obesity.

\section{Subjects and Methods}

2.1. Patients and Data Collection. This single-center retrospective study was performed in the general ICU of the University General Hospital of Patras (UGHP), Greece. UGHP is a tertiary hospital that accepts patients for the region of Western Greece, Peloponnese, and Ionian Islands and a population reaching one million people, whereas the ICU is separated in two compartments of ten and three beds, respectively. In the main compartment, two isolation and two semi-isolation beds are available. The medical records of all adult patients ( $\geq 18$ years) that were admitted from November 2011 to February 2014 were reviewed until their discharge from the ICU. The study was approved from the Ethical Committee of the University Hospital of Patras (number 571). The need for informed consent was waived because of the retrospective and observational design of the study according to European legislation.

Patient data (epidemiologic data, comorbidities, colonization/infection, antimicrobial administration, and ICU procedures) were prospectively collected and recorded in the ICU computerized database (Criticus $^{\mathrm{TM}}$, University of Patras, Greece). Severity scores of illness [APACHE II (Acute Physiology and Chronic Health Evaluation II), SAPS (Simplified Acute Physiology Score II), and SOFA (Sequential Organ Failure Assessment)] were calculated upon admission. Patients were included when they were aged 18 years or older. We excluded pregnant women and cardiac surgery patients. BMI was calculated for most of the patients upon ICU admission while nurses using a nonrigid measuring tape measured their height. According to the WHO criteria patients were categorized in five groups: underweight $\left(<18.5 \mathrm{~kg} / \mathrm{m}^{2}\right)$, normal weight $\left(18.5-24.9 \mathrm{~kg} / \mathrm{m}^{2}\right)$, overweight $\left(25-29.9 \mathrm{~kg} / \mathrm{m}^{2}\right)$, obese $\left(30-39.9 \mathrm{~kg} / \mathrm{m}^{2}\right)$, and morbidly obese $\left(\geq 40 \mathrm{~kg} / \mathrm{m}^{2}\right)$ [6]. Patients were categorized into the following groups according to the main reason for admission: trauma, spontaneous intracranial hemorrhage, sepsis, postoperative observation, respiratory insufficiency, and others ( $\operatorname{com} \alpha$, epilepsy, intoxication, etc.). Sepsis and septic shock were defined according to the Third International Consensus Definitions for Sepsis and Septic Shock [14].

2.2. Statistical Analysis. Statistical analysis was performed with SPSS version 22.0 software package (IBM SPSS Statistics for Mac, Version 22.0, Armonk, NY, USA). For the statistical analyses patients were further categorized in two groups: obese patients $\left(\mathrm{BMI} \geq 30 \mathrm{~kg} / \mathrm{m}^{2}\right)$ and nonobese patients (BMI $<30 \mathrm{~kg} / \mathrm{m}^{2}$ ). Categorical variables were analyzed by using the Fisher exact test or $\mathrm{chi}^{2}$ test while continuous variables were analyzed with Mann-Whitney $U$ test or one-way ANOVA, as appropriate. Three different analyses were performed resulting from a predefined analysis plan. The first one was aimed at determining factors that differ among obese and nonobese patients. The second one was aimed at detecting predictors of ICU mortality of patients that were septic upon admission and the third one was aimed at determining the factors that differ among obese and nonobese septic patients. Backward stepwise multiple logistic regression analysis used all those variables from the univariate analysis with $P<0.05$. In order to identify factors that were highly correlated, collinearity diagnostics were performed. No factors contributing to multicollinearity were revealed (tolerance $>0.2$ and VIF $<10$ for all the factors analyzed). All statistic tests were 2-tailed and $P<0.05$ was considered statistically significant.

\section{Results}

Of the 834 patients, 163 (19.5\%) were obese and among them $25(3.0 \%)$ were morbidly obese. Table 1 shows the univariate analysis of differences among obese and nonobese patients. Sixteen out of 38 factors were found to be statistically significant by univariate analysis (female gender, number of chronic diseases, diabetes mellitus, chronic obstructive disease, spontaneous intracranial hemorrhage, sepsis, ICU length of stay, ICU mortality, number of antibiotics administered, dialysis, enteral nutrition, KPC-Kp colonization, bloodstream infection and septic shock during ICU stay, KPC-Kp infection, and Candida infection during ICU stay). Multivariate analysis revealed that number of chronic diseases $(P<0.001$; OR 3.2; 95\% CI 2.6-3.9), bloodstream infection during ICU stay ( $P$ 0.033; OR 2.0; 95\% CI 1.1-3.7), and KPC-producing Klebsiella pneumoniae colonization during ICU stay $(P$ 0.005; OR 2.2; 95\% CI 1.3-3.7) were significantly associated with obesity, while nonobese patients suffer more frequently than obese patients from spontaneous intracranial hemorrhage (P 0.038; OR 0.22; 95\% CI 0.05-0.92).

Total ICU mortality was $22.5 \%$ (188 patients). Obese patients were characterized by increased ICU mortality, as compared to nonobese ones $(28.8 \%$ versus $21.0 \%$; $P$ 0.036). Table 2 shows the distribution of ICU patients according to the main reason for admission, BMI, and survival. Sepsis was the main condition of admission for which obese patients had statistically lower survival than normal weight subjects (76.3\% versus $43.7 \%$; $P$ 0.001).

In order to determine the predictors of mortality among septic patients upon ICU admission (125 patients), a second analysis was conducted (Table 3 ) by comparing survivors (58 patients) and nonsurvivors (67 patients). Univariate analysis revealed 12 statistically significant factors (number of chronic diseases, malignancy, cortisone use, obesity, SAPS II and SOFA score, parenteral nutrition, dialysis, meningitis, pneumonia, urinary-tract infection, and septic shock). Multivariate analysis revealed that SOFA score upon ICU admission ( $P$ 0.003; OR 1.3; 95\% CI 1.1-1.5), obesity $(P 0.014$; OR 5.3; 95\% CI 1.4-20.2), pneumonia ( $P$ 0.038; OR 3.5; $95 \%$ CI 1.1-11.3), and development of septic shock ( $P$ 0.015; OR 3.4; 95\% CI 1.3-9.1) were all independently associated with mortality, while urinary-tract infection $(P 0.010$; OR 0.06 ; 95\% CI 0.01-0.52) was associated with survival. Since obesity was an independent predictor of mortality among septic patients upon ICU admission, a further analysis was performed to assess the differences among obese and nonobese patients (Table 4). Twelve out of 34 factors were found to be statistically significant by univariate analysis (ICU length of 
TABLE 1: Univariate and multivariate analysis of factors that differ among obese and nonobese critically ill patients. $P$ depicts the univariate analysis results while $(*)$ denotes factors that differ in the multivariate analysis too $(P<0.001$ for number of chronic diseases, $P=0.033$ for bloodstream infection during ICU stay, $P 0.005$ for KPC-producing Klebsiella pneumoniae colonization during ICU stay, and $P$ 0.038 for spontaneous intracranial hemorrhage).

\begin{tabular}{|c|c|c|c|}
\hline Patient characteristics & Nonobese patients $(n=671)$ & Obese patients $(n=163)$ & $P$ \\
\hline \multicolumn{4}{|l|}{ Demographics } \\
\hline Age (years) & $56.3 \pm 19.8$ & $59.1 \pm 14.8$ & 0.095 \\
\hline Female gender & $129(32.2 \%)$ & $44(43.6 \%)$ & 0.035 \\
\hline Chronic diseases (number) & $0.7 \pm 0.9$ & $1.0 \pm 1.1$ & $0.001^{*}$ \\
\hline Diabetes mellitus & $82(12.2 \%)$ & $56(34.4 \%)$ & $<0.001$ \\
\hline Chronic obstructive pulmonary disease & $63(9.4 \%)$ & $39(23.9 \%)$ & $<0.001$ \\
\hline Chronic heart failure & $71(10.6 \%)$ & $20(12.3 \%)$ & 0.575 \\
\hline Chronic renal failure requiring dialysis & $38(5.7 \%)$ & $11(6.7 \%)$ & 0.580 \\
\hline Malignancy (solid organ or heamatologic one) & $162(24.1 \%)$ & $33(20.2 \%)$ & 0.305 \\
\hline Cortisone use (within last month of ICU admission) & $56(8.3 \%)$ & $9(5.5 \%)$ & 0.258 \\
\hline \multicolumn{4}{|l|}{ Reasons for admission } \\
\hline Spontaneous intracranial hemorrhage & $85(12.7 \%)$ & $2(1.2 \%)$ & $<0.001^{*}$ \\
\hline Sepsis & $87(13.0 \%)$ & $38(23.3 \%)$ & 0.001 \\
\hline Respiratory insufficiency & $63(9.4 \%)$ & $19(11.7 \%)$ & 0.463 \\
\hline Postoperative observation & $302(45.0 \%)$ & $71(43.6 \%)$ & 0.792 \\
\hline Trauma & $103(15.4 \%)$ & $22(13.5 \%)$ & 0.625 \\
\hline Others ${ }^{\mathrm{a}}$ & $31(4.6 \%)$ & $11(6.7 \%)$ & 0.316 \\
\hline \multicolumn{4}{|l|}{ Hospitalization data } \\
\hline Prior emergency surgery & $218(32.5 \%)$ & $49(30.1 \%)$ & 0.576 \\
\hline Prior abdominal surgery & $194(28.9 \%)$ & $49(30.1 \%)$ & 0.774 \\
\hline Prior hospitalization & $237(35.3 \%)$ & $57(35.0 \%)$ & 1.000 \\
\hline APACHE II Score upon admission & $13.3 \pm 7.5$ & $12.8 \pm 7.1$ & 0.679 \\
\hline SAPS II upon admission & $32.6 \pm 13.8$ & $33.8 \pm 13.1$ & 0.412 \\
\hline SOFA score upon admission & $6.4 \pm 3.8$ & $7.0 \pm 3.5$ & 0.051 \\
\hline ICU length of stay (days) & $8.8 \pm 11.4$ & $18.4 \pm 9.7$ & 0.004 \\
\hline ICU mortality & $141(21.0 \%)$ & $47(28.8 \%)$ & 0.036 \\
\hline \multicolumn{4}{|l|}{ ICU data } \\
\hline Cortisone & $225(33.5 \%)$ & $64(29.3 \%)$ & 0.170 \\
\hline Antibiotics administered (number) & $2.4 \pm 1.6$ & $2.9 \pm 2.2$ & 0.036 \\
\hline Mean antibiotic use per day & $1.9 \pm 1.0$ & $2.1 \pm 1.2$ & 0.088 \\
\hline Tracheostomy & $183(27.3 \%)$ & $55(33.7 \%)$ & 0.122 \\
\hline Dialysis & $27(4.0 \%)$ & $16(9.8 \%)$ & 0.005 \\
\hline Parenteral nutrition & $110(16.4 \%)$ & $38(23.3 \%)$ & 0.051 \\
\hline Enteral nutrition & $183(27.3 \%)$ & $59(36.2 \%)$ & 0.027 \\
\hline Number of invasive catheters ${ }^{\mathrm{b}}$ & $0.8 \pm 0.9$ & $1.0 \pm 1.4$ & 0.253 \\
\hline \multicolumn{4}{|l|}{ Colonization/infection data } \\
\hline KPC-Kp colonization during ICU stay & $134(20.0 \%)$ & $52(31.9 \%)$ & $0.002^{*}$ \\
\hline Days until colonization & $9.0 \pm 3.1$ & $9.6 \pm 3.6$ & 0.583 \\
\hline VRE colonization during ICU stay & $24(3.6 \%)$ & $8(4.9 \%)$ & 0.493 \\
\hline Bloodstream infection during ICU stay & $61(9.1 \%)$ & $35(21.5 \%)$ & $<0.001^{*}$ \\
\hline Septic shock during ICU stay & $91(13.6 \%)$ & $45(27.6 \%)$ & $<0.001$ \\
\hline KPC-Kp infection during ICU stay & $29(4.3 \%)$ & $19(11.7 \%)$ & 0.001 \\
\hline Candida infection during ICU stay & $9(1.3 \%)$ & $7(4.3 \%)$ & 0.022 \\
\hline
\end{tabular}

Data are number (\%) of patients or mean \pm SD.

ICU: intensive care unit; APACHE II: Acute Physiology and Chronic Health Evaluation II; SAPS: Simplified Acute Physiology Score II; SOFA: Sequential Organ Failure Assessment; KPC-Kp: KPC-producing K. pneumoniae; VRE: vancomycin-resistant Enterococcus.

${ }^{a}$ Coma, epilepsy, myocardial infarction, and intoxication.

${ }^{\mathrm{b}}$ All patients after ICU admission were intubated and mechanically ventilated and were continuously monitored with a central venous catheter, an arterial catheter, and a urinary catheter. Number of catheters does not include the aforementioned ones. 


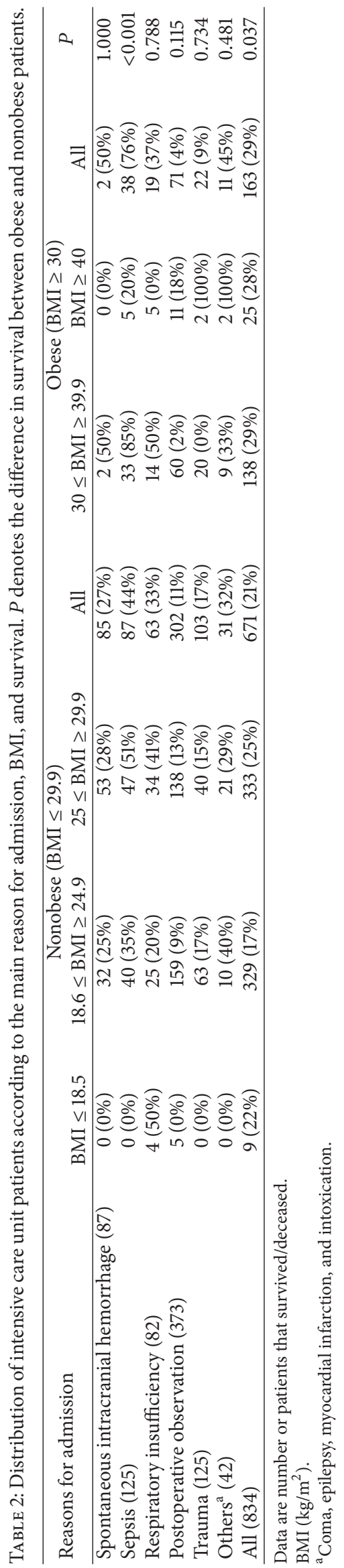


TABLE 3: Univariate and multivariate analysis ofpredictors of mortality among septic patients upon intensive care unit admission. $P$ depicts the univariate analysis results while $(*)$ denotes factors that differ in the multivariate analysis too $(P 0.003$ for SOFA score upon admission, $P 0.014$ for obesity, $P 0.038$ for pneumonia, and $P 0.015$ for septic shock).

\begin{tabular}{|c|c|c|c|}
\hline Patient characteristics & Survivors $(n=58)$ & Nonsurvivors $(n=67)$ & $P$ \\
\hline \multicolumn{4}{|l|}{ Demographics } \\
\hline Age (years) & $59.0 \pm 19.7$ & $61.0 \pm 17.1$ & 0.603 \\
\hline Male gender & $28(57.1 \%)$ & $27(57.4 \%)$ & 1.000 \\
\hline Chronic diseases (number) & $0.9 \pm 1.0$ & $1.6 \pm 1.2$ & $<0.001$ \\
\hline Diabetes mellitus & $10(17.2 \%)$ & $17(25.4 \%)$ & 0.286 \\
\hline Chronic obstructive pulmonary disease & $12(20.7 \%)$ & $16(23.9 \%)$ & 0.830 \\
\hline Chronic heart failure & $8(13.8 \%)$ & $9(13.4 \%)$ & 1.000 \\
\hline Chronic renal failure requiring dialysis & $5(8.6 \%)$ & $4(6.0 \%)$ & 0.732 \\
\hline Malignancy (solid organ or heamatologic one) & $2(3.4 \%)$ & $13(19.4 \%)$ & 0.006 \\
\hline Cortisone use (within last month of ICU admission) & $3(5.2 \%)$ & $13(19.4 \%)$ & 0.029 \\
\hline Obesity & $9(15.5 \%)$ & $29(43.3 \%)$ & $0.001^{*}$ \\
\hline \multicolumn{4}{|l|}{ Hospitalization data } \\
\hline Prior emergency surgery & $14(24.1 \%)$ & $15(22.4 \%)$ & 0.835 \\
\hline Prior abdominal surgery & $17(29.3 \%)$ & $17(25.4 \%)$ & 0.689 \\
\hline Prior hospitalization & $36(62.1 \%)$ & $49(73.1 \%)$ & 0.249 \\
\hline APACHE II Score upon admission & $16.9 \pm 7.2$ & $19.1 \pm 8.4$ & 0.323 \\
\hline SAPS II upon admission & $39.8 \pm 12.9$ & $46.3 \pm 12.8$ & 0.007 \\
\hline SOFA score upon admission & $8.1 \pm 3.1$ & $9.9 \pm 4.2$ & $0.037^{*}$ \\
\hline ICU length of stay (days) & $14.5 \pm 7.2$ & $17.0 \pm 17.4$ & 0.216 \\
\hline \multicolumn{4}{|l|}{ ICU data } \\
\hline Cortisone & $32(55.2 \%)$ & $48(71.6 \%)$ & 0.064 \\
\hline Antibiotics administered (number) & $3.5 \pm 1.8$ & $3.9 \pm 1.9$ & 0.149 \\
\hline Mean antibiotic use per day & $2.8 \pm 0.9$ & $2.9 \pm 0.9$ & 0.539 \\
\hline Tracheostomy & $25(43.1 \%)$ & $32(47.8 \%)$ & 0.719 \\
\hline Number of invasive catheters $^{\mathrm{a}}$ & $0.8 \pm 1.4$ & $1.1 \pm 1.5$ & 0.072 \\
\hline Parenteral nutrition & $11(19.0 \%)$ & $24(35.8 \%)$ & 0.046 \\
\hline Enteral nutrition & $27(46.6 \%)$ & $35(52.2 \%)$ & 0.592 \\
\hline Dialysis & $3(5.2 \%)$ & $13(19.4 \%)$ & 0.029 \\
\hline \multicolumn{4}{|l|}{ Infection upon admission data } \\
\hline \multicolumn{4}{|l|}{ Site of infection } \\
\hline Meningitis & $8(13.8 \%)$ & $2(3.0 \%)$ & 0.044 \\
\hline Pneumonia & $22(37.9 \%)$ & $40(59.7 \%)$ & $0.020^{*}$ \\
\hline Intra-abdominal infection & $13(22.4 \%)$ & $14(20.9 \%)$ & 1.000 \\
\hline Urinary-tract infection & $12(20.7 \%)$ & $3(4.5 \%)$ & 0.011 \\
\hline Skin and soft tissue infection & $3(5.2 \%)$ & $7(10.4 \%)$ & 0.337 \\
\hline Bloodstream infection & $6(10.3 \%)$ & $16(23.9 \%)$ & 0.060 \\
\hline Septic shock & $8(13.8 \%)$ & $47(70.1 \%)$ & $<0.001^{*}$ \\
\hline \multicolumn{4}{|l|}{ Colonization data } \\
\hline KPC-Kp colonization during ICU stay & $20(34.5 \%)$ & $21(35.8 \%)$ & 1.000 \\
\hline VRE colonization during ICU stay & $5(8.6 \%)$ & $6(9.0 \%)$ & 1.000 \\
\hline
\end{tabular}

Data are number $(\%)$ of patients or mean \pm SD.

ICU: intensive care unit; APACHE II: Acute Physiology and Chronic Health Evaluation II; SAPS: Simplified Acute Physiology Score II; SOFA: Sequential Organ Failure Assessment; KPC-Kp: KPC-producing K. pneumoniae; VRE: vancomycin-resistant Enterococcus.

${ }^{a}$ All patients after ICU admission were intubated and mechanically ventilated and were continuously monitored with a central venous catheter, an arterial catheter, and a urinary catheter. Number of catheters does not include the aforementioned catheters. 
TABLE 4: Univariate and multivariate analysis fordifference among obese and nonobese septic patients upon intensive care unit admission. $P$ depicts the univariate analysis results while $(*)$ denotes factors that differ in the multivariate analysis too $(P 0.002$ for mortality, $P 0.042$ for bloodstream infection upon ICU admission, and P 0.001 for KPC-producing K. pneumoniae colonization during ICU stay).

\begin{tabular}{|c|c|c|c|}
\hline Patient characteristics & Nonobese $(n=87)$ & Obese $(n=38)$ & $P$ \\
\hline \multicolumn{4}{|l|}{ Demographics } \\
\hline Age (years) & $60.5 \pm 19.5$ & $59.2 \pm 15.2$ & 0.464 \\
\hline Male gender & $41(62.1 \%)$ & $14(46.7 \%)$ & 0.185 \\
\hline Chronic diseases (number) & $0.9 \pm 0.9$ & $1.1 \pm 1.1$ & 0.242 \\
\hline Diabetes mellitus & $16(18.2 \%)$ & $11(28.9 \%)$ & 0.238 \\
\hline Chronic obstructive pulmonary disease & $17(19.5 \%)$ & $11(28.9 \%)$ & 0.253 \\
\hline Chronic heart failure & $12(13.8 \%)$ & $5(13.2 \%)$ & 1.000 \\
\hline Chronic renal failure requiring dialysis & $8(9.2 \%)$ & $1(2.6 \%)$ & 0.274 \\
\hline Malignancy (solid organ or heamatologic one) & $9(10.3 \%)$ & $6(15.8 \%)$ & 0.385 \\
\hline Cortisone use (within last month of ICU admission) & $10(11.5 \%)$ & $6(15.8 \%)$ & 0.564 \\
\hline \multicolumn{4}{|l|}{ Hospitalization data } \\
\hline Prior emergency surgery & $18(20.7 \%)$ & $11(28.9 \%)$ & 0.360 \\
\hline Prior abdominal surgery & $21(24.1 \%)$ & $13(34.2 \%)$ & 0.278 \\
\hline Prior hospitalization & $59(67.8 \%)$ & $26(68.4 \%)$ & 1.000 \\
\hline APACHE II Score upon admission & $18.7 \pm 7.8$ & $16.2 \pm 7.8$ & 0.169 \\
\hline SAPS II upon admission & $42.8 \pm 13.3$ & $44.3 \pm 13.2$ & 0.658 \\
\hline SOFA score upon admission & $9.0 \pm 3.9$ & $9.2 \pm 3.8$ & 0.786 \\
\hline ICU length of stay (days) & $11.9 \pm 12.6$ & $24.9 \pm 26.4$ & $<0.001$ \\
\hline Mortality & $38(43.7 \%)$ & $29(76.3 \%)$ & $0.001^{*}$ \\
\hline \multicolumn{4}{|l|}{ ICU data } \\
\hline Cortisone & $49(56.3 \%)$ & $31(81.6 \%)$ & 0.008 \\
\hline Antibiotics administered (number) & $3.4 \pm 1.5$ & $4.9 \pm 2.5$ & $<0.001$ \\
\hline Mean antibiotic use per day & $2.8 \pm 0.9$ & $3.1 \pm 0.9$ & 0.005 \\
\hline Tracheostomy & $31(35.6 \%)$ & $26(68.4 \%)$ & 0.001 \\
\hline Number of invasive catheters ${ }^{\mathrm{a}}$ & $0.5 \pm 0.8$ & $1.9 \pm 2.1$ & $<0.001$ \\
\hline Parenteral nutrition & $19(21.8 \%)$ & $16(42.1 \%)$ & 0.030 \\
\hline Enteral nutrition & $37(42.5 \%)$ & $25(65.8 \%)$ & 0.020 \\
\hline Dialysis & $6(6.9 \%)$ & $10(26.3 \%)$ & 0.007 \\
\hline \multicolumn{4}{|l|}{ Colonization/infection data } \\
\hline \multicolumn{4}{|l|}{ Site of infection } \\
\hline Meningitis & $9(10.3 \%)$ & $1(2.6 \%)$ & 0.280 \\
\hline Pneumonia & $44(50.6 \%)$ & $18(47.4 \%)$ & 0.846 \\
\hline Intra-abdominal infection & $15(17.2 \%)$ & $12(31.6 \%)$ & 0.098 \\
\hline Urinary-tract infection & $13(14.9 \%)$ & $2(5.3 \%)$ & 0.148 \\
\hline Skin and soft tissue infection & $6(6.9 \%)$ & $4(10.5 \%)$ & 0.490 \\
\hline Bloodstream infection (primary or secondary) & $10(11.5 \%)$ & $12(31.6 \%)$ & $0.010^{*}$ \\
\hline Septic shock & $34(39.1 \%)$ & $21(55.3 \%)$ & 0.123 \\
\hline \multicolumn{4}{|l|}{ Colonization data } \\
\hline KPC-Kp colonization during ICU stay & $21(24.1 \%)$ & $23(60.5 \%)$ & $<0.001^{*}$ \\
\hline VRE colonization during ICU stay & $7(8.0 \%)$ & $4(10.5 \%)$ & 0.734 \\
\hline
\end{tabular}

Data are number (\%) of patients or mean \pm SD.

ICU: intensive care unit; APACHE II: Acute Physiology and Chronic Health Evaluation II; SAPS: Simplified Acute Physiology Score II; SOFA: Sequential Organ Failure Assessment; KPC-Kp: KPC-producing K. pneumoniae; VRE: vancomycin-resistant Enterococcus.

${ }^{a}$ All patients after ICU admission were intubated and mechanically ventilated and were continuously monitored with a central venous catheter, an arterial catheter, and a urinary catheter. Number of catheters does not include the aforementioned catheters. 


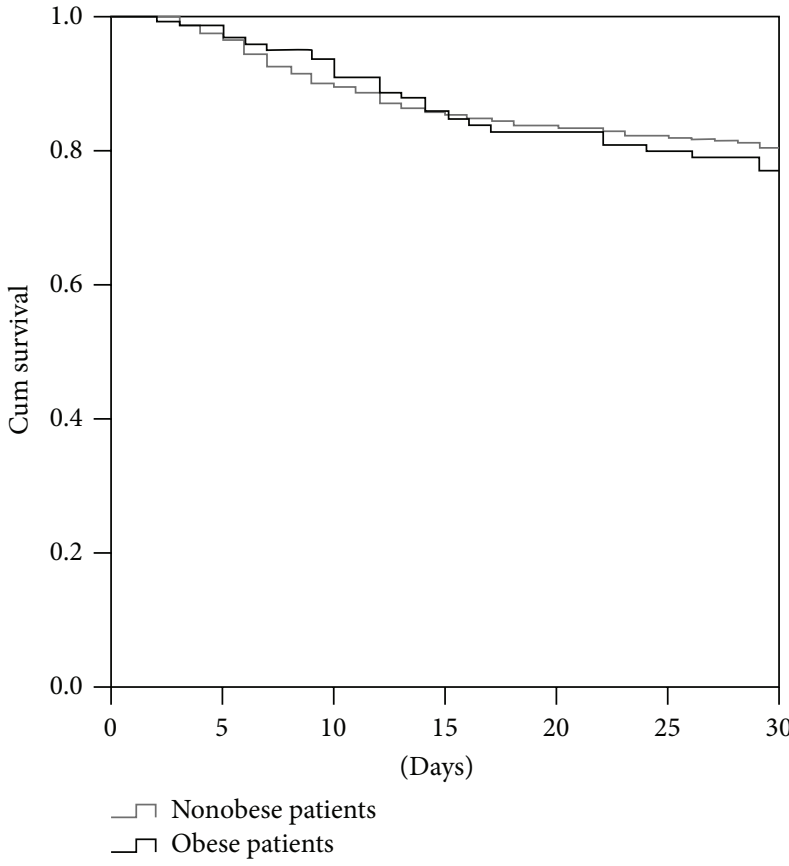

(a)

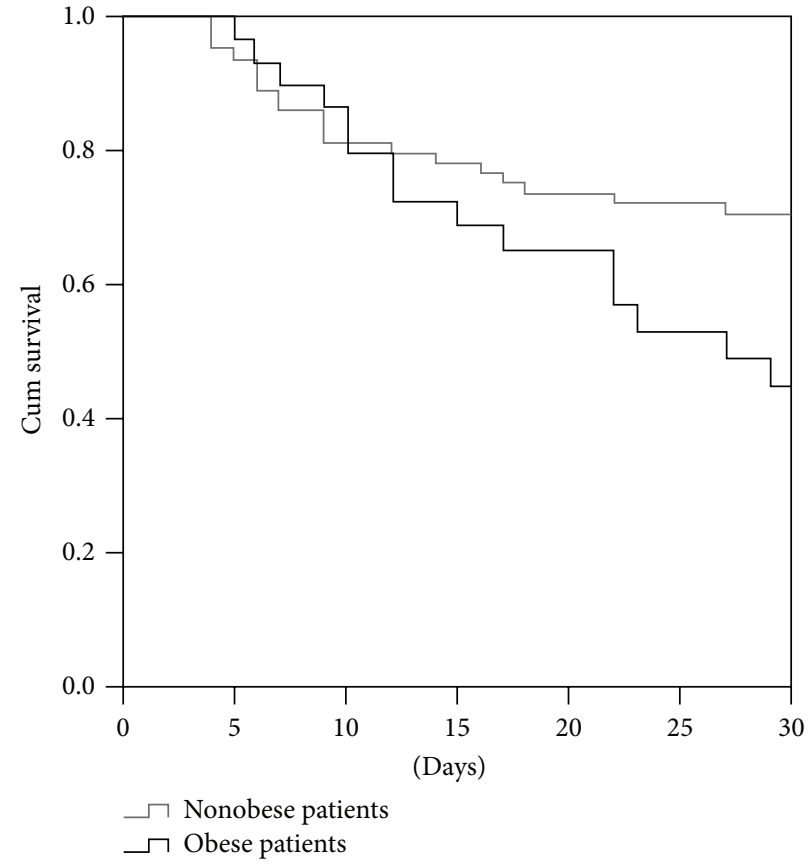

(b)

FIGURE 1: Kaplan-Meier curves of 30-day survival probability according to the presence of obesity of (a) of all patients and (b) septic patients upon ICU admission.

stay, mortality, cortisone use, number of antibiotics administered, mean antibiotic use per day, tracheostomy, number of invasive catheters, parenteral and enteral nutrition, dialysis, bloodstream infection, and KPC-Kp colonization during ICU stay). In multivariate analysis mortality ( $P$ 0.002; OR 4.9; 95\% CI 1.8-13.2), bloodstream infection upon ICU admission (P 0.042; OR 3.1; 95\% CI 1.0-9.0), and KPC-producing $K$. pneumoniae colonization during ICU stay $(P$ 0.001; OR 2.1; 95\% CI 1.3-3.2) were significantly associated with obese septic patients. According to Kaplan-Meier curves the 30day survival probability is lower in obese septic patients compared to nonobese ones (Figure 1).

\section{Discussion}

The percentages of overweight (39.9\%) and obese (19.5\%) patients admitted to the ICU were similar to that reported from previous prevalence studies in Greek population [3-5]. Obese patients suffered more often from chronic diseases as compared to nonobese patients, especially diabetes mellitus and chronic obstructive pulmonary disease, while male gender was more common among nonobese patients admitted to our ICU $[2,4]$. An interesting finding of our study, reported for the first time, was that spontaneous intracranial (subarachnoid or intraparenchymal) hemorrhage was independently more common in nonobese patients. This contradicts the fact that obesity is a well-known risk factor for nontraumatic brain hemorrhage [15]. The low percentage of obese patients with brain hemorrhage in our study probably does not reflect the reality since, in the present study, only patients admitted to the ICU were included and not patients hospitalized in other hospital wards such as the neurosurgery department. Moreover, this is a retrospective study and further studies are needed in order to elucidate this association. The relatively high percentage of patients with intracranial hemorrhage admitted to the ICU can be explained by the fact that our hospital is the only tertiary hospital, with a neurosurgical department, in a region of one million people. All patients with intracranial hemorrhage diagnosed in other hospitals are transferred to our hospital.

In our study obese critically ill patients had lower ICU survival as compared to nonobese patients, in contrary to the results of previous studies that showed that obesity has a beneficial effect on ICU mortality $[9,10]$. This phenomenon, which is known as "obesity paradox", has no apparent physiological explanation [11]. In a large cohort study Abhyankar et al. found that overweight and obese patients had higher survival rate both thirty days and one year after ICU admission [10]. In our study only obese patients admitted for sepsis presented higher mortality rate compared to nonobese patients, while no difference in outcome was observed in obese patients with other admission reasons. Like previous studies our study also showed that obesity did not influence mortality in patients admitted to the ICU for postoperative observation (nonobese $10.6 \%$ versus obese $4.2 \%, P 0.115$ ) [16]. Moreover, the same observation was made for patients admitted after emergency surgery $(25.2 \%$ versus $34.4 \%, P$ 0.376) [17].

Obesity has been identified as a risk factor for the development of nosocomial and community-acquired infections [18], but the relation between obesity and infection in critically ill patients is unclear [19]. In our study obesity was associated with infection upon admission and bacteremia 
during ICU stay. Dossett et al. have also shown that the rate of primary or catheter related bloodstream infection was significantly more common in obese as compared to nonobese patients [20]. Obesity has been seldom identified as a risk factor for colonization by multidrug resistant pathogens [21]. It is noteworthy in the present study that obese patients during ICU stay became more commonly colonized by KPC-producing K. pneumoniae, which resulted in higher incidence of infections provoked by the same pathogen. KPC-producing K. pneumoniae is prevalent in Greek ICUs causing infections associated with increased mortality [22]. Even though it can be argued that the high colonization rate among obese patients may be due to the prolonged length of stay of these patients (18.4 versus 8.8 days; $P$ 0.004), we found that the length of stay until colonization by the aforementioned pathogen did not differ among obese and nonobese patients ( 9.6 versus 9.0 days; $P$ 0.583), indicating that the length of stay plays no role in the colonization incidence. The higher rate of colonization and subsequent infection by KPC-producing K. pneumoniae may explain the increased antibiotic administration among critically ill obese patients (Table 1), especially those used for the treatment of aforementioned infection, such as colistin, aminoglycosides, and tigecycline [23]. Obese patients received more commonly antifungal treatment, which can be explained by the increased length of stay of obese patients in the ICU and the higher rate of Candida infection. The latter is due to the fact that obesity predisposes to Candida species colonization which makes obese patients susceptible to candidemia [23].

Our study shows higher mortality rates in obese septic patients upon ICU admission as compared to nonobese ones. Even though obesity can be expected to be a predictor of mortality among septic patients, this topic remains a subject of considerable debate $[12,24]$. A previous retrospective study of patients with septic shock found that obesity was associated with lower mortality, an association that could be influenced by the difference of age and comorbidities among groups [12]. Our study contradicts the results of the aforementioned study. The true effect of obesity on increased mortality, in our study, can be also supported by the fact that obese and nonobese septic patients had the same age and severity of illness upon ICU admission as it is depicted by APACHE II Score, SAPS II, and SOFA score while they also suffered from the same number of comorbidities and had similar rates of septic shock upon ICU admission. In the subgroup of patients admitted with pneumonia (66 patients), mortality was higher among obese patients (56.8\% versus $83.3 \%$; $P$ 0.078). On the contrary a previous study showed a protective effect of obesity on mortality from community-acquired pneumonia [25]. This discrepancy of results can be explained by the fact that in our cohort study some cases of pneumonia were nosocomial and only severe cases of pneumonia warranting ICU admission were included.

A great percentage of patients (373/834) were admitted to the ICU for postoperative observation. There were similar rates of postoperative admission between obese and nonobese patients while the types of surgeries were the same. The operations included were abdominal, cardiovascular, neurosurgical, and orthopedic and no difference concerning the type of surgery was observed.

Our study has several limitations. Although we included all patients admitted to the ICU during a 28-month period, this is a single-center retrospective study. A second limitation of our study would be the fact that although the study included critically ill patients from a region of one million people, with high rates of multidrug resistant pathogens, our data cannot be extrapolated to patients worldwide. Finally, measurement of height or weight in recumbent patients is associated with errors arising from the presence of numerous attachments or volume depletion or overload.

\section{Conclusion}

In our study prevalence of obesity among critically ill patients reflects prevalence of obesity of Greek population. Obesity was associated with higher incidence of infection and lower incidence of nontraumatic brain hemorrhage while, among patients with sepsis upon ICU admission, obesity was associated with higher mortality. The obesity paradox (lower mortality) was not observed in Greek critically ill patients probably due to the fact that obese ICU patients develop more commonly infections, especially by KPC-producing K. pneumoniae, which are associated with reduced survival. More studies are needed in order to evaluate the relationship between obesity and colonization or infection by multidrug resistant pathogens.

\section{Abbreviations}

ICU: Intensive care unit

LOS: Length of stay.

\section{Competing Interests}

All authors state that they have no conflict of interests to report.

\section{Acknowledgments}

The study was supported by funding of the Anaesthesiology and Intensive Care Medicine, School of Medicine, University of Patras, Greece.

\section{References}

[1] M. L. Roditis, E. S. Parlapani, T. Tzotzas, M. Hassapidou, and G. E. Krassas, "Epidemiology and predisposing factors of obesity in Greece: From the Second World War until today," Journal of Pediatric Endocrinology and Metabolism, vol. 22, no. 5, pp. 389405, 2009.

[2] E. Koloverou, D. B. Panagiotakos, C. Pitsavos et al., "10-Year incidence of diabetes and associated risk factors in Greece: the ATTICA study (2002-2012)," The Review of Diabetic Studies, vol. 11, no. 2, pp. 181-189, 2014.

[3] M. Ng, T. Fleming, M. Robinson et al., "Global, regional, and national prevalence of overweight and obesity in children and adults during 1980-2013: a systematic analysis for the Global 
Burden of Disease Study 2013," The Lancet, vol. 284, no. 9945, pp. 766-781, 2014.

[4] G. N. Koukoulis, C. Sakka, F. Katsaros et al., "High rates of obesity prevalence in adults living in Central Greece: data from the ARGOS study," Hormones, vol. 9, no. 3, pp. 253-262, 2010.

[5] G. Andrikopoulos, D. Richter, D. Sakellariou et al., "High prevalence and diminished awareness of overweight and obesity in a mediterranean population. An alarming call for action," Open Cardiovascular Medicine Journal, vol. 6, no. 1, pp. 141-146, 2012.

[6] World Health Organization, Obesity and Overweight. Fact Sheet No. 311, World Health Organization, Geneva, Switzerland, 2015, http://www.who.int/mediacentre/factsheets/fs311/en/ index.html.

[7] S. A. Nasraway Jr., M. Albert, A. M. Donnelly, R. Ruthazer, S. A. Shikora, and E. Saltzman, "Morbid obesity is an independent determinant of death among surgical critically ill patients," Critical Care Medicine, vol. 34, no. 4, pp. 964-970, 2006.

[8] N. Bercault, T. Boulain, K. Kuteifan, M. Wolf, I. Runge, and J.-C. Fleury, "Obesity-related excess mortality rate in an adult intensive care unit: a risk-adjusted matched cohort study," Critical Care Medicine, vol. 32, no. 4, pp. 998-1003, 2004.

[9] Y. Sakr, C. Elia, L. Mascia et al., "Being overweight or obese is associated with decreased mortality in critically ill patients: a retrospective analysis of a large regional Italian multicenter cohort," Journal of Critical Care, vol. 27, no. 6, pp. 714-721, 2012.

[10] S. Abhyankar, K. Leishear, F. M. Callaghan, D. DemnerFushman, and C. J. McDonald, "Lower short- and long-term mortality associated with overweight and obesity in a large cohort study of adult intensive care unit patients," Critical Care, vol. 16, no. 6, article R235, 2012.

[11] R. N. Dickerson, "The obesity paradox in the ICU: real or not?" Critical Care, vol. 17, no. 3, article 154, 2013.

[12] Y. M. Arabi, S. I. Dara, H. M. Tamim et al., "Clinical characteristics, sepsis interventions and outcomes in the obese patients with septic shock: an international multicenter cohort study," Critical Care, vol. 17, no. 2, article R72, 2013.

[13] S. Utzolino, C. M. Ditzel, P. K. Baier, U. T. Hopt, and M. F. Kaffarnik, "The obesity paradox in surgical intensive care patients with peritonitis," Journal of Critical Care, vol. 29, no. 5, pp. 887.el-887.e5, 2014.

[14] M. Singer, C. S. Deutschman, C. W. Seymour et al., "The third international consensus definitions for sepsis and septic shock (Sepsis-3)," The Journal of the American Medical Association, vol. 315, no. 8, pp. 801-810, 2016.

[15] B. E. Zacharia, Z. L. Hickman, B. T. Grobelny et al., "Epidemiology of Aneurysmal Subarachnoid Hemorrhage," Neurosurgery Clinics of North America, vol. 21, no. 2, pp. 221-233, 2010.

[16] R. Gupta, M. Villa, E. Agaba et al., "The effect of body mass index on the outcome of critically ill surgical patients," Journal of Parenteral and Enteral Nutrition, vol. 37, no. 3, pp. 368-374, 2013.

[17] P. Ferrada, R. J. Anand, A. Malhotra, and M. Aboutanos, "Obesity does not increase mortality after emergency surgery," Journal of Obesity, vol. 2014, Article ID 492127, 3 pages, 2014.

[18] M. E. Falagas and M. Kompoti, "Obesity and infection," The Lancet Infectious Diseases, vol. 6, no. 7, pp. 438-446, 2006.

[19] R. Huttunen and J. Syrjänen, "Obesity and the risk and outcome of infection," International Journal of Obesity, vol. 37, no. 3, pp. 333-340, 2013.
[20] L. A. Dossett, L. A. Dageforde, B. R. Swenson et al., "Obesity and site-specific nosocomial infection risk in the intensive care unit," Surgical Infections, vol. 10, no. 2, pp. 137-142, 2009.

[21] M. Papadimitriou-Olivgeris, M. Christofidou, F. Fligou et al., "The role of colonization pressure in the dissemination of colistin or tigecycline resistant KPC-producing Klebsiella pneumoniae in critically ill patients," Infection, vol. 42, no. 5, pp. 883890, 2014.

[22] M. Papadimitriou-Olivgeris, M. Marangos, M. Christofidou et al., "Risk factors for infection and predictors of mortality among patients with KPC-producing Klebsiella pneumoniae bloodstream infections in the intensive care unit," Scandinavian Journal of Infectious Diseases, vol. 46, no. 9, pp. 642-648, 2014.

[23] M. Papadimitriou-Olivgeris, A. Spiliopoulou, F. Fligou et al., "Association of KPC-producing Klebsiella pneumoniae colonization or infection with Candida isolation and selection of non-albicans species," Diagnostic Microbiology and Infectious Disease, vol. 80, no. 3, pp. 227-232, 2014.

[24] R. L. Smith, T. W. Chong, T. L. Hedrick et al., "Does body mass index affect infection-related outcomes in the intensive care unit?" Surgical Infections, vol. 8, no. 6, pp. 581-588, 2007.

[25] V. F. Corrales-Medina, J. Valayam, J. A. Serpa, A. M. Rueda, and D. M. Musher, "The obesity paradox in community-acquired bacterial pneumonia," International Journal of Infectious Diseases, vol. 15, no. 1, pp. e54-e57, 2011. 


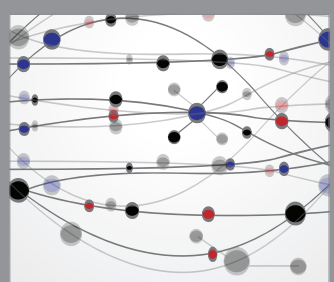

The Scientific World Journal
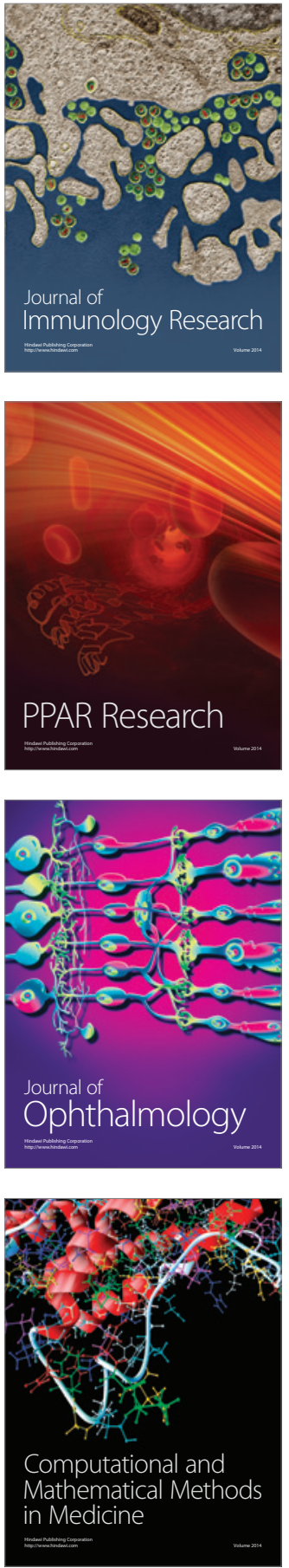

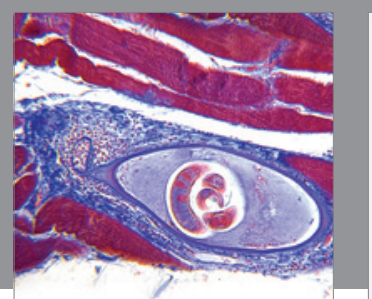

Gastroenterology Research and Practice

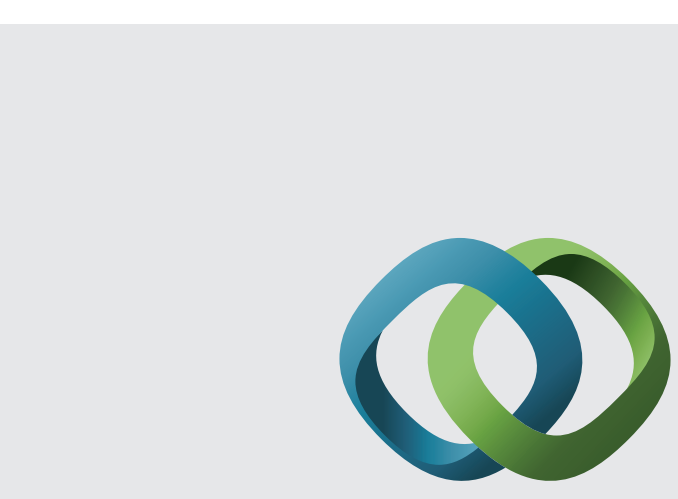

\section{Hindawi}

Submit your manuscripts at

http://www.hindawi.com
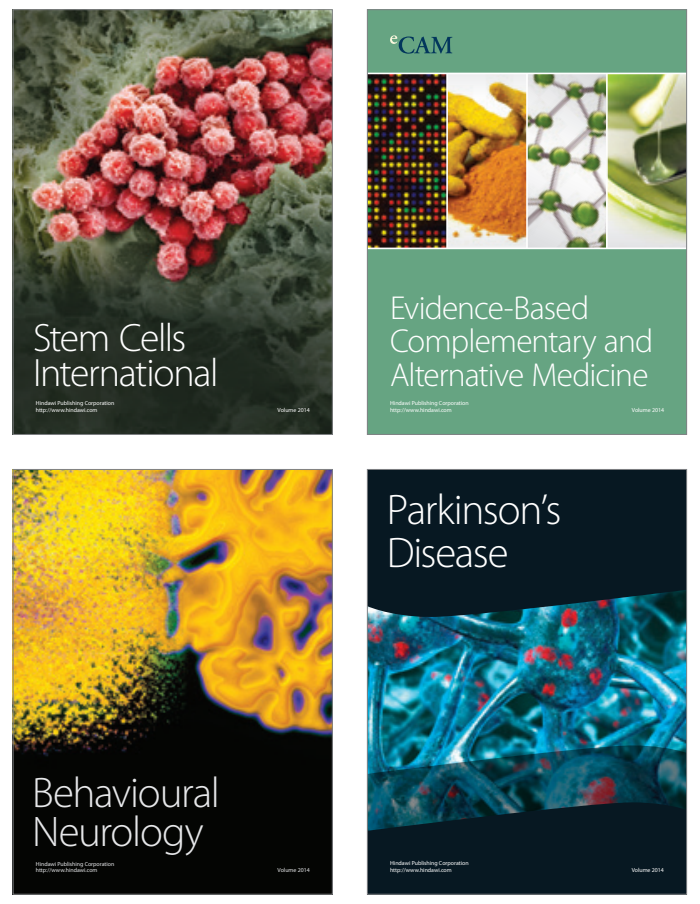
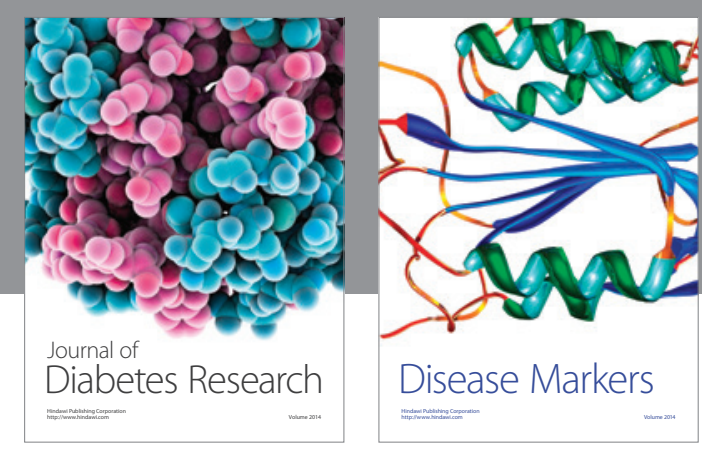

Disease Markers
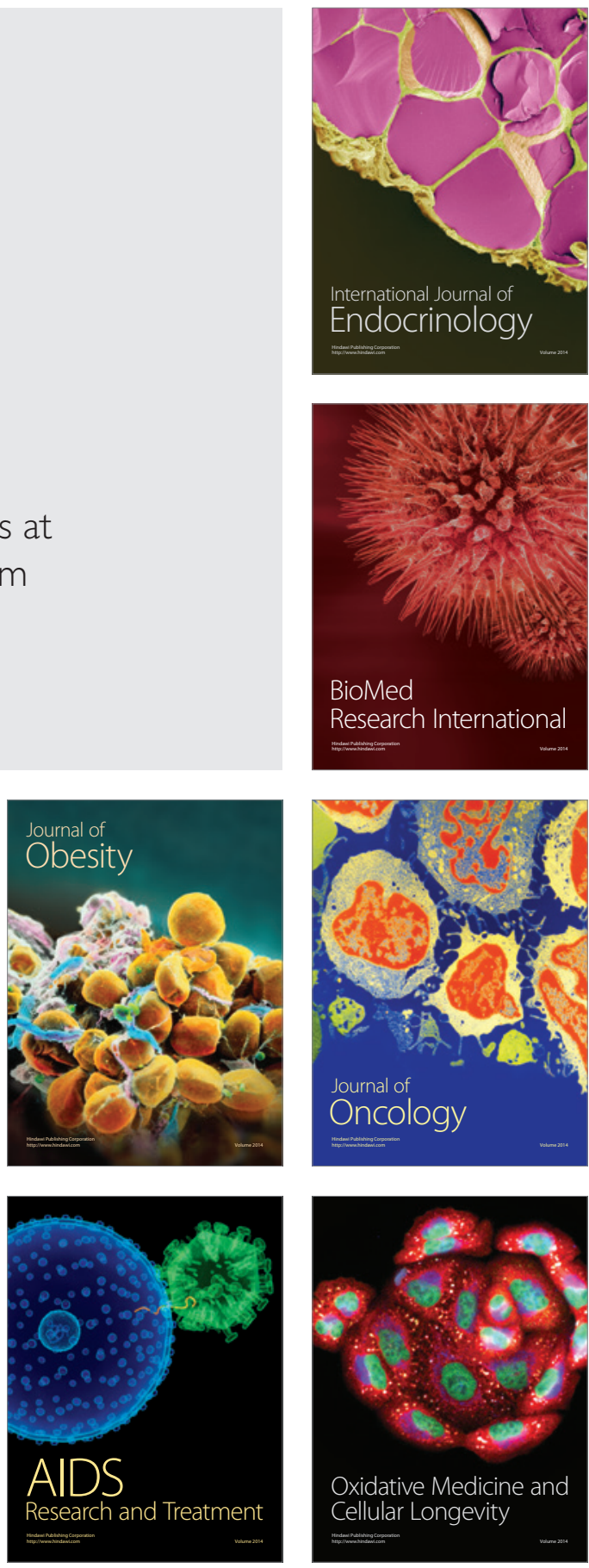